INTERNATIONAL JOURNAL OF RESEARCHES IN BIOSCIENCES, AGRICULTURE AND TECHNOLOGY (C) VISHWASHANTI MULTIPURPOSE SOCIETY (Global Peace Multipurpose Society) R. No. MH-659/13(N) www.vmsindia.org

\title{
SPECTRAL BEHAVIOR OF DIFFERENT SPECIES BY TIME SERIES TEMPORAL PROFILES FOR ESTIMATION OF CROP ACREAGE FORECASTING
}

\author{
R. R. Nagpure \\ Sevadal Mahila Mahavidyalaya \& Research Academy, Umrer Road, Nagpur, (M.S.), India \\ raghoba09@yahoo.com
}

\begin{abstract}
:
Crop identification with remote sensing data facilitates only when crop has sufficiently grown-up. However, forecasting of crop at sowing stage would require use of weather data and information on economic factors controlling the farmer's response. Time series remote sensing data is being used to monitor the crop through its growing period. Vegetation indices and weather parameter derived from surface and satellite observations are used to develop the crop growth monitoring system. Present paper deals with the methodology adopted for discrimination of special crop using NDVI time series derived from multi-date Satellite data. Study area covers the dominant Rabi crop growing districts of Maharashtra state. Multi-date satellite data for rabi season of $2013-14$ have been used. Two-stage classification of this dataset by unsupervised Iterative Self Organizing Data Analysis Technique, labeling of classes based on temporal spectral profiles of Rabi Sorghum and other competing crops and decision rule based integration were followed to generate final classified image. This hybrid classification technique takes advantage of inherent clustering tendency of vegetation and non-vegetation classes in feature space with temporal dimension added to it in terms of Normalized Difference Vegetation Index (NDVI) time series data. It also makes use of signatures of known crop classes for labeling the clusters. Satellite data, being coarse, intricacies involved in its spatial accuracy are not covered in this study as it aims at acreage estimation only. Special Rabi crop acreage estimated by this approach for the state is 3721.12 ('000') ha. The only purpose of multi-forecasting at state level stage serves well by adopting this hybrid approach.

Keywords: Satellite data, Temporal profiles, Crop Acreage Forecasting.
\end{abstract}

\section{Introduction:}

Agriculture is the backbone of Indian economy, contributing about 40 percent towards the Gross National Product (GNP) thereby providing livelihood to about 70 percent of the population. Reliable, accurate and timely information on types of crops grown, acreage, crop yield and crop growth conditions are vital components for the planners engaged in formulating and implementing appropriate policies pertaining to prices of agricultural commodities, strengthening country's food security, distribution system and import/export formulations, contributing to GNP. The agricultural crop production is usually estimated as a product of area under the crop and the average yield per unit area of the crop. With the advent of Remote Sensing (RS) technology and spectral indices based classification techniques, have opened new vistas of enhancing systems, revolving around crop discrimination, identification, classification and mapping. Multi-date, multi-spectral data in the form of several vegetation indices have been utilized for crop identification and area estimation world wide. Crop growth profiles, derived using spectral indices, have been modeled by (Badhwar 1980) and by (Christ and Malila 1980) using multi-date Landsat - MSS data. Spectral yield models for corn and soybean based on growth profile for some selected time periods are studied by (Boatwright et. al. 1988).

Digital classification of RS data, is a method by which labels or class identifiers are attached to the pixels based on the characteristics spectrally homogeneous in nature. These characteristics are measurements of their spectral response in different wavebands along with other attributes or temporal signatures. The most commonly used classification methodologies used in remote sensing are unsupervised procedures such as Iterative Self Organizing Data Analysis Technique (ISODATA) and supervised methods, the most popular of which is the Maximum Likelihood (MXL) algorithm, where precise filtering of the information registered on imageries is carried out with image analysis algorithms.

Radiometric value of each pixel with predefined number of clusters are effectively utilized in the ISODATA clustering algorithm where, cluster attractors shifts the cluster mean values in a way that the majority of the pixels belongs to a cluster gets agglomerated. The user in this case interacts with the procedure at the beginning indicating the number of the predefined clusters to be created and the iterations to be carried out. User decides which class represents which surface objects and merges or rejects the classes with non-realistic representatives (Manakos et al., 2000). There exist some ground features that are spectrally similar and can't be separated using ISODATA clustering. Researchers have used various classifiers for classifying the RS data for different applications (Tateishi et al., 1991; 
Friedl and Brodley, 1997; Hastings, 1997; Friedl et al., 1999). Multi-date IRS WiFS and AWiFS data have been used for various agricultural applications including cropping pattern change monitoring, crop yield modelling and crop classification (Rajak et al., 2002; Rajak et al., 2005; Oza et al., 2008).

Rabi sorghum is a semi-arid tropical crop cultivated extensively in Central India. Rabi sorghum grown in Western and Marathwada region of Maharashtra State alone contribute to about $67 \%$ of all India acreage and $55 \%$ of all India rabi sorghum production. During rabi season, sorghum is the major crop and is cultivated in large contiguous areas. Monitoring of crop condition, development of crop growth profile and prediction of yield based on remotely sensed multi-spectral data hold great promise. In the present study, a hybrid technique of multi-date RS data classification based on twostage ISODATA clustering and visual classification has been presented. It takes advantages of the natural groupings of pixels based on their spectral properties by way of ISODATA clustering and discrimination of spectrally similar classes by way of visual vector polygon analysis.

\section{Materials and Methods:}

The study was carried out over a part of Maharashtra state, that belongs to major rabi sorghum growing states of India. It includes 19 rabi sorghum growing districts (Figure 1). The major rabi crops grown in the area are rabi sorghum, wheat, gram etc. Biannual cash crop, sugarcane is also one of the competing crop present in the field during the rabi season. Most of the rabi crops in the region are grown on the residual soil moisture, except, irrigated areas in the command. The major source of irrigation are river, canals. The data set used in this study comprised of multi-date Satellite data, in-season ground truth information, sampling grid of $5 \times 5$ $\mathrm{km}$, district / state boundaries vector polygons etc. The IRS satellite data covering rabi seasons of 2013-14 (October November/December/Jan/Feb) were used.

The methodology consists of multi-date satellite data analysis using time series temporal profile, adopting hybrid approach for rabi sorghum acreage estimation. The crop grows, the reflectance in the Near Infrared (NIR) begins to increase due to an increase in the spongy mesophyll structure and decreases in the red spectral region due to an increased absorption by chlorophyll. Ground based experiments under controlled environment indicate that spectral index of the crop canopy at any given point of time is a measure of the total green biomass. This description when extended to the entire temporal domain of the crop growth reveals that, maximum leaf area index during peak vegetative growth is analogous to peak value of the crop growth profile and rate of senescence can be an average slope of the profile in post-heading time domain. The flow diagram of the methodology is shown in Figure 2 and elaborated in the following steps.

\section{Geo-referencing and Image Stacking:}

The multi-date satellite data set was geo-referenced by image-to-image registration using tile $\left(40 \times 4^{0}\right)$ wise master image in TM (Transverse Mercatar) projection. Geo-referenced RS data are stacked to prepare single image. Multi-date RS data analysis needs a high degree of accuracy for image-to image registrations. All the registrations were carried out to meet the accuracy requirement in terms of root mean square error less than 0.5 pixels.

\section{Radiometric Normalisation:}

The raw digital numbers (DN) images from satellite data were converted to radiance images using the calibration coefficients. The relationship between $\mathrm{DN}$ and radiance $(\mathrm{L})$ is given by:

\section{$\mathrm{L}=\mathrm{Lmin}+\mathrm{DN} . .(\mathrm{Lmax}-\mathrm{Lmin}) / \mathrm{DNmax}$}

Where $\mathrm{Lmin}=$ minimum radiance Lmax $=$ saturation radiance / maximum radiance DNmax $=$ Radiometric resolution

The values of Lmax and Lmin for different spectral bands of the two sensors are given in Table 1 . The levels of DNmax were taken as 1024 (10 bit data i.e. 0 to 1023 ) for this data.

\section{Preparation of Reference Temporal Spectral Profiles (RTSP) and scaling of NDVI:}

The intensity or digital number (DN) value, of each visible red band is subtracted from the NIR band on a pixel-by-pixel basis. The value is then divided by the sum of the two NDVI derived from the radiance values of two spectral bands, is defined as:

$$
\text { LNIR - LR }
$$

$$
\text { NDVI }=\text { - } \mathrm{LNIR}+\mathrm{LR}
$$

Scaled NDVI $=100+100 \times(\mathrm{NDVI})$

where,

LNIR is radiance value for NIR band

$L R$ is radiance value for Red band.

The result of NDVI consists of continuous floating-point data that ranges from -1 to 1 , including decimal and negative values, 
but for simplicity, it was scaled in the range of 0 to 200, multiplying by 100 and adding 100 .

The geographical locations of field surveys are then transferred to the images. Based on the details collected during field visits, training windows are marked on the images of acquisition date closed to field observation date. Multi date signatures and vegetation indices values are extracted from these training windows.These signatures provides RTSPs of rabi sorghum and other competing crops with non-crop features.

\section{Data Load Reduction of Multi-date RS Dataset:}

Non Crop Mask (NCM) has been prepared using already available information on wasteland, forest, slope, built-up areas etc. To reduce the number of data pixels to be subjected for ISODATA clustering, all pixels belonging to non-agricultural areas were masked out. It was carried out by multiplying NDVI stacked data with NCM image. The NCM image contains 1 and 0 for all pixels belonging to crops and non-agriculture, respectively. The output multi-layer dataset is then used for further processing in ISODATA clustering stage1 and stage-II.

\section{ISODATA Clustering and Labelling (Stage-I and stage-II):}

Pixels are analysed beginning with the upper left corner of the image and going left to right, block by block. The spectral distance between the candidate pixels and each cluster mean is calculated. The ISODATA clustering method uses spectral distance as in sequential method, but iteratively classifies the pixels, redefines the criteria for each class, and classifies again, so that the spectral distance pattern in the data gradually emerges. The pixels are assigned to the cluster whose mean is the closest. The ISODATA function creates an output image file with a thematic raster layer and/or a signature file as a result of the clustering. The signature file so generated has been used to generate Temporal Spectral Profile (TSPs) for each class. Each of these TSPs of classes is then compared with all available RTSPs of crop of the study area. The TSP of cluster class is assigned to the RTSP with which it matches most in temporal domain within threshold limit of RTSP. Matching of TSPs with RTSPs is carried out by visual observation and with analyst's expert knowledge of the region.

All the inherent crop classes present in the multi-date image may not be discriminated in the first attempt of ISODATA clustering itself. Consequently, there may remain some clusters that may not be assigned to any of the RTSPs. Such clusters are put under "Mix crop" class. Also due to lack of RTSPs some cluster classes may remain "unclassified" in the classified image. The pixels belonging to "Mix crop" class and "unclassified" class are further fed to ISODATA clustering (stage-II) and same procedure is reapeted as in the case of ISODATA (stage-I). Final output of ISODATA stage-I and Stage-II are stored separately.

Visual Interpretation of Residual Pixels:

Unclassified pixels and/or pixels which interpreter can not assign to any crop class with confidence using available RTSPs even after ISODATA Stage-I and Stage-II, are smoothened using median filter and are converted to vector coverage. This vector coverage is then overlaid on multi-dates RS images. Polygon are visually analysed and are labelled accordingly. In case of spatially uniform polygons, TSP of pixels belonging to these polygons are visually compared with RTSPs and the polygons attributes are modified accordingly.

\section{Integration of classified images/vector and} Acreage Estimation:

The output of ISODATA stage-I and stage-II analysis and visually interpreted polygons are integrated to create the final classified image. This integration is based on simple decision rules that take into account the class label of each pixel in three different layers. For extracting rabi sorghum acreages, a stratified random sampling approach is adopted. It is based on the sampling fraction of $15 \%$ from a $5 \times 5 \mathrm{~km}$ grid overlaid on the study area. Rabi sorghum pixels in each of the grid is estimated by histogram method and clubbed into 4 strata namely; A, B, C, and D. Using the said statistical sampling technique number of rabi sorghum pixels are estimated and are converted into acreage by multiplying with spatial resolution of AWiFS sensor. Strata wise rabi sorghum acreage are further aggregated to calculate the acreage of rabi sorghum in study district. This figure is then extrapolated for state level acreage estimation.

\section{Results and Discussion:}

Temporal profile indicates, the spectral pattern of rabi sorghum, which is clearly different from that of other competing and noncrop classes. This wide difference in temporal profiles of non-crop classes from that of crop classes helps ISODATA clustering in separating non-crop clusters from crop clusters. The analyst could easily assign the different land use classes to the unknown clusters using basic knowledge of signatures and comparing the 
spatial distribution of unknown classes with respect to False Colour Composite images of different dates. Multi-date satellite images spanning through November 2013 to February 2014 are shown in (Figure 3).
Rabi sorghum acreage integrated through classification procedures, aggregated from different stratas and extrapolated to state is 3721.12 ('000') ha.

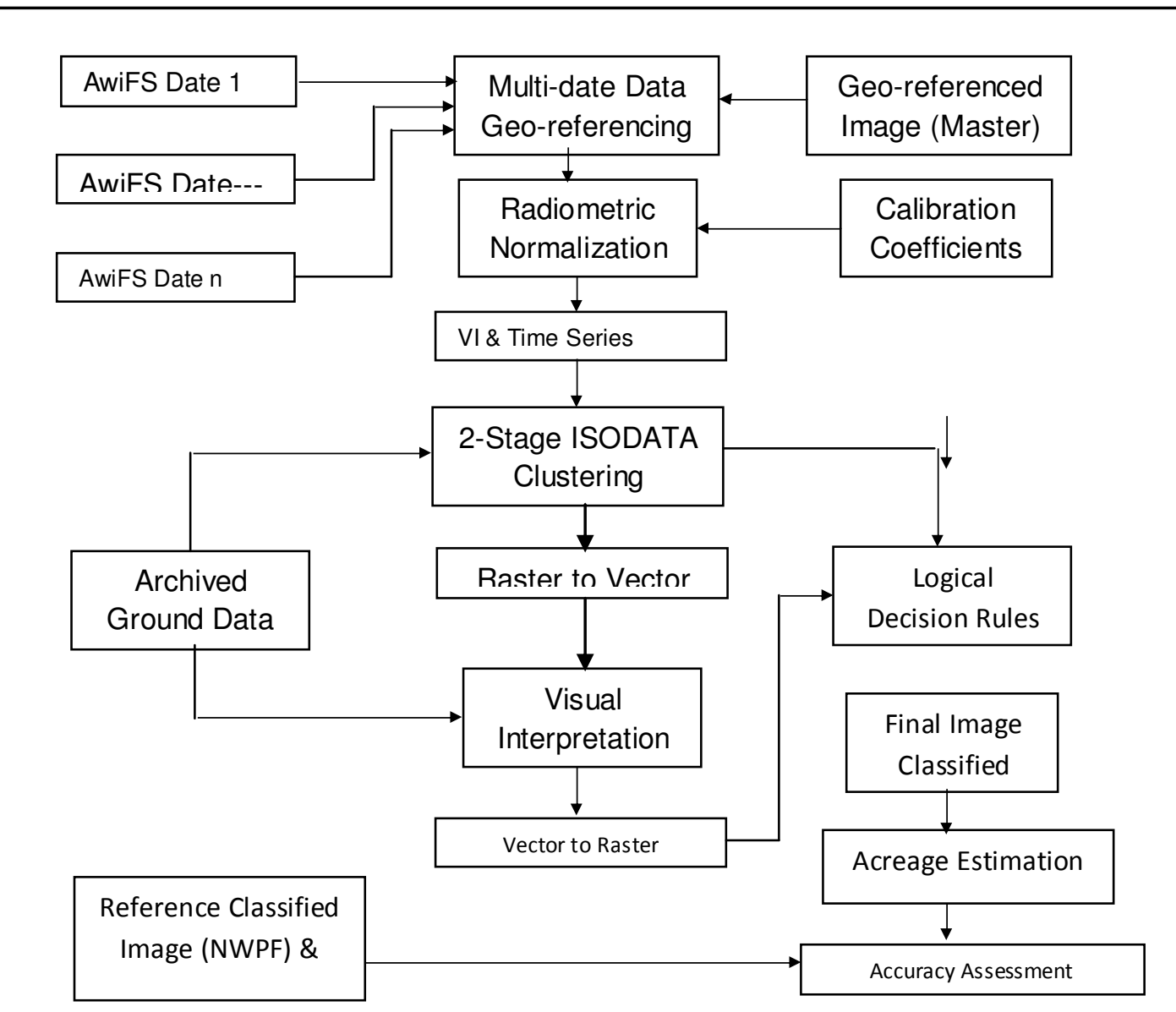

Figure. 2- Flow chart of methodology adopted in the study

Table 1: Calibration coefficients used for computing radiance values

\begin{tabular}{|l|l|l|l|l|}
\hline Sensor & $\begin{array}{l}\text { Spectral } \\
\text { Band }\end{array}$ & $\begin{array}{l}\text { Band } \lambda \text { Range } \\
(\boldsymbol{\mu m})\end{array}$ & $\mathbf{L}_{\text {max }^{\#}}$ & $\mathbf{L}_{\text {min }}$ \\
\hline AWiFS & Green & $0.52-0.59$ & $53.0^{*}$ & 0 \\
\hline AWiFS & Red & $0.62-0.68$ & $47.0^{*}$ & 0 \\
\hline AWiFS & NIR & $0.77-0.86$ & $31.5^{*}$ & 0 \\
\hline AWiFS & SWIR & $1.55-1.70$ & $07.5^{*}$ & 0 \\
\hline
\end{tabular}

* Source: NRSA, 2003

\# Lmax in mw/sq.cm/str/micron

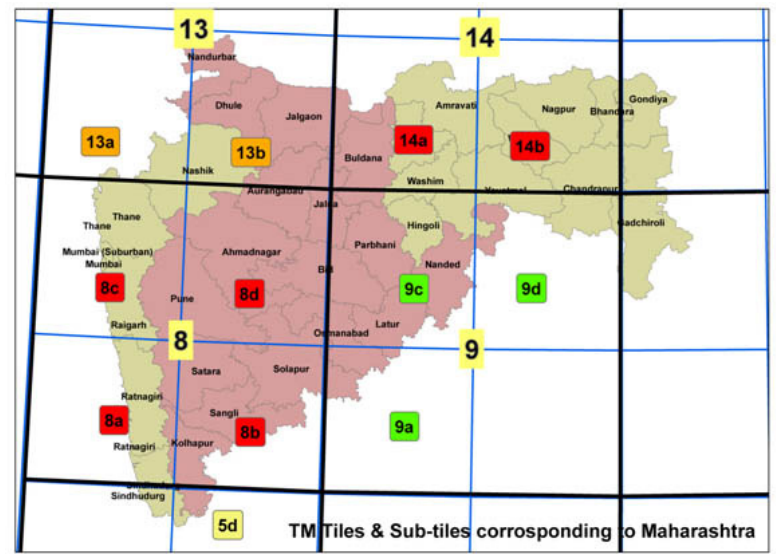

Figure 1: Districts covered under study overlaid by TM tiles and sub-tiles 


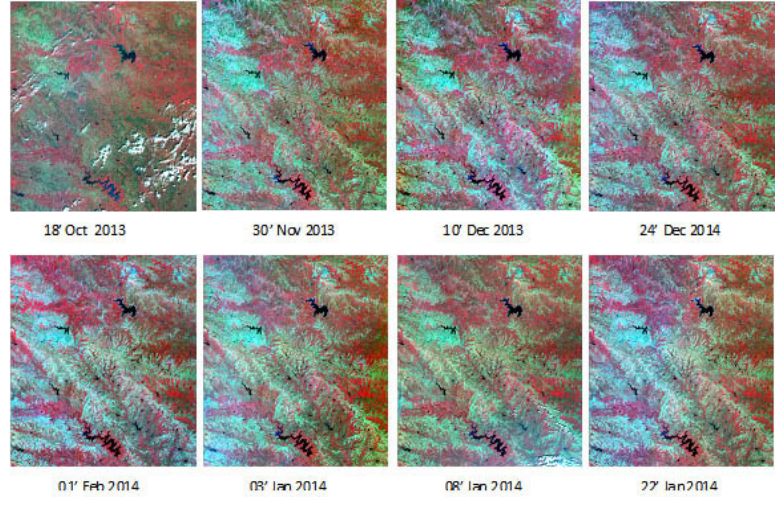

Figure. 2- Multi-date Satellite Data Conclusion:

The hybrid technique demonstrates the utility of multi-date RS data classification, integrated two-stage ISODATA clustering and visual classification of unknown vector polygons. The two-stage ISODATA clustering yielded in a classified image having 93 to 98 percent pure-class pixels and 2 to 7 percent mixed-class pixels. The labeling of unknown classes in first stage clustering was based on analyst's knowledge of basic signatures of land use / land cover classes. The pixels belonging to non-crop classes were masked out before the second stage clustering. The labeling of unknown classes of the second stage clustering was based on visual matching of temporal NDVI patterns of unknown classes with those of known crops in the study area. The mixed classes after second stage clustering were vectorised and then class polygons were manually edited for assigning pure crop classes to them. The final classified image was prepared by integrating the pure classes from second stage clustering output and edited vector output. The rabi sorghum acreage estimated from the final classified image for the study area comprising thirteen districts of Maharashtra state was estimated to be 3721.12 ('000') ha. More than 95 percent pixels classified as rabi sorghum were correctly classified using the hybrid technique. It is observed that many linear features like canals and roads were correctly classified as non-sorghum classes in the present study. Further, as remote sensing methods regularize continuous landscapes into a grid of equal sized and regularly spaced data in the form of pixels, it is anticipated that there will be some degree of dependency between pixels, most likely in the form of positive spatial autocorrelation. Such dependence has potentially a dual impact on the analysis of image data. Such a holistic approach where multi-date RS data fed to rigorous and repetitive analysis makes it conceptually sounder than those methods, involving single-date spectral indexes which rely solely on spectral data within a narrow critical period maximum vegetative growth phase.

\section{Reference:}

1. Badhwar, G. D. 1980. Crop emergence date determination from spectral data. Photogrammetric Engineering and Remote Sensing, 46:369-377.

2. Boatwright, G. O., Badhwar, G. D. and Johnson, W.R. (1988), An AVHRR spectral based yield model for corn and soybeans. Final Report, Project No. 8103, USDA-ARS. Beltsville, Maryland, USA.

3. Christ, E.P. and Malila, w.A. (1980), Temporal spectral analysis of technique for vegetation applications of Landsat, Proceeding of the 14th Symposium on Remote Sensing of Environment, held at San Jose, Costa Rica, 23-30 April, pp. 10311041.

4. Fried1, M. A. and Brodley, C.E. 1997. Decision tree classification of land cover from remotely sensed data. Remote Sensing of Environment. 61, 399-409.

5. Friedl, M. A., Brodley, C. E., and Strahler, A.H. 1999. Maximizing land cover classification accuracies produced by decision tree at continental to global scales. IEEE Transactions on Geoscience and Remote Sensing. 37, 969-977.

6. Hastings, David A. 1997. Land cover classification: some new techniques, new source data. Proceedings, 18th Asian Conference on Remote Sensing. Tokyo, Asian Association of Remote Sensing. Pp. JS-2-1 to JS-2-6.

7. Manakos, I., Schneider, T. and U. Ammer. 2000. A comparison between the ISODATA and the eCognition classification methods on basis of field data. Poster at the XIXth ISPRS Congress, Amsterdam, Presentation number: TP VII-09-22, Netherlands.

8. Oza, M. P., Pandya, M. R. and Rajak, D. R. 2008. Evaluation and use of Resourcesat-I data for agricultural applications. International Journal of Applied Earth Observation and Geoinformation 10, 194-205

9. Rajak, D. R., Oza, M. P., Bhagia, N. and Dadhwal, V. K. 2002. Monitoring Cropping Pattern Changes Using Multi-temporal WiFS Data. Geocarto International, 17(2), 51-56.

10. Rajak, D. R, Oza, M. P., Bhagia, N. and Dadhwal, V. K. 2005. Spectral Wheat Growth Profile in Punjab Using IRS WiFS Data. Journal of the Indian Society of Remote Sensing, 33(2): 345352.

11. Tateishi, R., K. Kajiwara, and T. Odajima. 1991. Global land cover classification by phenological methods using NOAA GVI data. AsianPacific Remote Sensing Journal. vol. 4, no. 1, pp. 41-50. 